International Mathematical Forum, 1, 2006, no. 1, 17-25

\title{
SOME NEW PARAMETRIC FAMILIES OF MULTIVARIATE COPULAS
}

\author{
Ali Dolati \\ Department of Statistics, Faculty of Science, Yazd University, \\ Yazd, 89195-741, Iran. \\ dolati50@yahoo.com \\ Manuel Úbeda-Flores \\ Departamento de Estadística y Matemática Aplicada, \\ Universidad de Almería, \\ Carretera de Sacramento s/n, La Cañada de San Urbano, \\ 04120 Almería, Spain. \\ mubeda@ual.es

\begin{abstract}
Recently, Rodríguez-Lallena and Úbeda-Flores [8] have introduced a class of bivariate copulas which generalizes some known families such as the Farlie-Gumbel-Morgenstern distributions. In this paper we present multivariate generalizations of this class studying symmetry and dependence concepts, measuring the dependence among the components of each class, and providing several examples.
\end{abstract}

Mathematics Subject Classification: 62H05, 62H20.

Keywords: Copulas, Dependence concepts, Measures of association, Ordering, Symmetry.

\section{Introduction}

Rodríguez-Lallena and Úbeda-Flores [8] describe a (one-parametric) family of bivariate copulas of the form

$$
C_{\lambda}(u, v)=u v+\lambda f(u) g(v), \quad(u, v) \in[0,1]^{2},
$$

where $f$ and $g$ are two non-zero absolutely continuous functions such that $f(0)=f(1)=g(0)=g(1)=0$, and the admissible range of the parameter $\lambda$ is $[-1 / \max (\alpha \gamma, \beta \delta),-1 / \min (\alpha \delta, \beta \gamma)]$, where $\alpha=\inf \left\{f^{\prime}(u): u \in A\right\}$, 
$\beta=\sup \left\{f^{\prime}(u): u \in A\right\}, \gamma=\inf \left\{g^{\prime}(v): v \in B\right\}$ and $\delta=\sup \left\{g^{\prime}(v): v \in B\right\}$, with $A=\left\{u \in[0,1]: f^{\prime}(u)\right.$ exists $\}$ and $B=\left\{v \in[0,1]: g^{\prime}(v)\right.$ exists $\}$. This class of copulas provides a method for constructing bivariate distributions with a variety of dependence structures and generalizes several known families such as the Farlie-Gumble-Morgenstern (FGM) distributions and other (see, for instance, [1], [2], [3] and [8]). In this paper we provide procedures to construct parametric families of multivariate distributions which generalize (1). For statistical applications, we refer to [4] and [5].

Let $n \geq 2$ be a natural number. An $n$-copula is the restriction to $[0,1]^{n}$ of a continuous multivariate distribution function whose univariate margins are uniform on $[0,1]$. The importance of copulas is described in the following result: Let $\mathbf{X}=\left(X_{1}, \cdots, X_{n}\right)$ be a random vector with joint distribution function $H$ and respective margins $F_{1}, \cdots, F_{n}$. Then there exists an $n$-copula $C$ (which is uniquely determined on Range $F_{1} \times \cdots \times$ Range $\left.F_{n}\right)$ such that $H\left(x_{1}, \cdots, x_{n}\right)=$ $C\left(F_{1}\left(x_{1}\right), \cdots, F_{n}\left(x_{n}\right)\right)$ for all $\mathbf{x}=\left(x_{1}, \cdots, x_{n}\right)$ in $[-\infty, \infty]^{n}$. If $F_{1}, \cdots, F_{n}$ are continuous, then $C$ is unique. In the sequel, we shall suppose that $\mathbf{X}$ is continuous. For a complete review, see [6].

If $\mathbf{u}=\left(u_{1}, \cdots, u_{n}\right)$ is a point in $[0,1]^{n}, \Pi^{n}(\mathbf{u})=\prod_{i=1}^{n} u_{i}$ denotes the $n$ copula of independent continuous random variables. Let $\mathbf{X}>\mathbf{x}$ denote the component-wise inequality, and let $\mathbf{1}$ be an $n$-dimensional vector of $1^{\prime} s$. If $\mathbf{U}$ is a vector of uniform $[0,1]$ random variables with $n$-copula $C$, then $\hat{C}(\mathbf{u})=$ $P[\mathbf{1}-\mathbf{U}<\mathbf{u}]$ and $\bar{C}(\mathbf{u})=P[\mathbf{U}>\mathbf{u}]$ are, respectively, the survival copula and the survival function associated with $C$. Given two $n$-copulas $C_{1}$ and $C_{2}$, let $C_{1} \leq C_{2}$ denote the inequality $C_{1}(\mathbf{u}) \leq C_{2}(\mathbf{u})$ for all $\mathbf{u} . C_{1}$ is more positive lower (respectively, upper) orthant dependent (PLOD) (respectively, PUOD) than $C_{2}$ if $C_{1} \geq C_{2}$ (respectively, $\bar{C}_{1} \geq \bar{C}_{2}$ ). $C_{1}$ is more positive orthant dependent (POD) than $C_{2}$ if both $C_{1} \geq C_{2}$ and $\bar{C}_{1} \geq \bar{C}_{2}$ hold (the corresponding negative concepts are defined by reversing the sense of the inequalities). A parametric family of $n$-copulas $\left\{C_{\theta}\right\}, \theta \in \mathbb{R}$, is positively ordered when $\theta_{1} \leq \theta_{2}$ implies that $C_{\theta_{1}} \leq C_{\theta_{2}}$. If $C$ is an $n$-copula, $C$ is radially symmetric if $C=\hat{C}$; and $C(\pi \mathbf{u})$ denotes the $n$-copula given by any permutation $\pi$ of $\mathbf{u}$.

The population versions of two non-parametric measures of multivariate association between components of a continuous random vector $\mathbf{U}$ with associated $n$-copula $C$ are two multivariate generalizations of Kendall's tau and Spearman's rho, which are given by

$$
\begin{gathered}
\tau_{n}(C)=\frac{1}{2^{n-1}-1}\left(2^{n} \int_{[0,1]^{n}} C(\mathbf{u}) \mathrm{d} C(\mathbf{u})-1\right) \text { and } \\
\rho_{n}(C)=\frac{n+1}{2^{n}-(n+1)}\left[2^{n-1}\left(\int_{[0,1]^{n}} C(\mathbf{u}) \mathrm{d} \Pi^{n}(\mathbf{u})+\int_{[0,1]^{n}} \Pi^{n}(\mathbf{u}) \mathrm{d} C(\mathbf{u})\right)-1\right],
\end{gathered}
$$

respectively (see [7]). 


\section{New parametric families of $n$-copulas}

\subsection{A first family}

A first one-parameter multivariate extension of the class of copulas given by $(1)$ is

$$
C_{\theta}(\mathbf{u})=\prod_{i=1}^{n} u_{i}+\theta \prod_{i=1}^{n} f_{i}\left(u_{i}\right), \quad \mathbf{u} \in[0,1]^{n}
$$

where $\theta \in \mathbb{R}$ and $f_{i}, 1 \leq i \leq n$, are $n$ non-zero absolutely continuous functions such that $f_{i}(0)=f_{i}(1)=0$. Note that all the $k$-dimensional margins, $2 \leq k<n$, are $\Pi^{k}$. The density function of $(2)$ is $c_{\theta}(\mathbf{u})=1+\theta \prod_{i=1}^{n} f_{i}^{\prime}\left(u_{i}\right)$, whose parameter $\theta$ has the admissible range $-1 / \sup _{\mathbf{u} \in D^{+}}\left(\prod_{i=1}^{n} f_{i}^{\prime}\left(u_{i}\right)\right) \leq \theta \leq$ $-1 / \inf _{\mathbf{u} \in D^{-}}\left(\prod_{i=1}^{n} f_{i}^{\prime}\left(u_{i}\right)\right)$, where $D^{-}=\left\{\mathbf{u} \in[0,1]^{n}: \prod_{i=1}^{n} f_{i}^{\prime}\left(u_{i}\right)<0\right\}$ and $D^{+}=$ $\left\{\mathbf{u} \in[0,1]^{n}: \prod_{i=1}^{n} f_{i}^{\prime}\left(u_{i}\right)>0\right\}$.

The survival function and the survival $n$-copula associated with the $n$ copula $C_{\theta}$ are given by $\bar{C}_{\theta}(\mathbf{u})=\prod_{i=1}^{n}\left(1-u_{i}\right)+(-1)^{n} \theta \prod_{i=1}^{n} f_{i}\left(u_{i}\right)$ and $\hat{C}_{\theta}(\mathbf{u})=$ $\prod_{i=1}^{n} u_{i}+(-1)^{n} \theta \prod_{i=1}^{n} f_{i}\left(1-u_{i}\right)$, respectively, for every $\mathbf{u}$ in $[0,1]^{n}$.

The expressions $\tau_{n}\left(C_{\theta}\right)$ and $\rho_{n}\left(C_{\theta}\right)$ for a member of the family (2) are given by $\tau_{n}\left(C_{\theta}\right)=2 I_{1} /\left(2^{n-1}-1\right)$ and $\rho_{n}\left(C_{\theta}\right)=(n+1) I_{1} /\left[2^{n}-(n+1)\right]$, where $I_{1}=\left(1+(-1)^{n}\right) 2^{n-1} \theta \prod_{i=1}^{n} \int_{0}^{1} f_{i}(t) \mathrm{d} t$. Observe that for this class of $n$-copulas we have the following relationship:

$$
\rho_{n}\left(C_{\theta}\right)=\frac{(n+1)\left(2^{n-1}-1\right) \tau_{n}\left(C_{\theta}\right)}{2\left(2^{n}-n-1\right)} .
$$

Let $\left\{C_{\theta}\right\}$ be the corresponding family of $n$-copulas given by (2). Then, $\left\{C_{\theta}\right\}$ is positively ordered if and only if $\prod_{i=1}^{n} f_{i}\left(u_{i}\right) \geq 0$ for all $\mathbf{u}$ in $[0,1]^{n}$.

Let $C_{\theta_{1}}(\mathbf{u})=\prod_{i=1}^{n} u_{i}+\theta_{1} \prod_{i=1}^{n} f_{i}\left(u_{i}\right)$ and $C_{\theta_{2}}(\mathbf{u})=\prod_{i=1}^{n} u_{i}+\theta_{2} \prod_{i=1}^{n} g_{i}\left(u_{i}\right)$ be two $n$-copulas. Then, $C_{\theta_{1}}$ is more PLOD (respectively, PUOD) than $C_{\theta_{2}}$ if and only if $\theta_{1} \prod_{i=1}^{n} f_{i}\left(u_{i}\right) \geq \theta_{2} \prod_{i=1}^{n} g_{i}\left(u_{i}\right)$ (respectively, $(-1)^{n} \theta_{1} \prod_{i=1}^{n} f_{i}\left(1-u_{i}\right) \geq$ $\left.(-1)^{n} \theta_{2} \prod_{i=1}^{n} g_{i}\left(1-u_{i}\right)\right)$ 
Let $A$ and $B$ be two non-empty disjoint subsets of $\{1, \cdots, n\}$ such that $|A \cup B|=n$, and let $\mathbf{U}_{A}=\left(U_{i}: i \in A\right)$ and $\mathbf{U}_{B}=\left(U_{j}: j \in B\right)$ denote subsets of the coordinates of the random vector $\mathbf{U} . \mathbf{U}_{B}$ is left tail decreasing in $\mathbf{U}_{A}\left(\operatorname{LTD}\left(\mathbf{U}_{B} \mid \mathbf{U}_{A}\right)\right)$ if $P\left(\mathbf{U}_{B} \leq \mathbf{u}_{B} \mid \mathbf{U}_{A} \leq \mathbf{u}_{A}\right)$ is non-increasing in each component of $\mathbf{u}_{A}$ for all $\mathbf{u}_{B} ; \mathbf{U}_{B}$ is right tail increasing in $\mathbf{U}_{A}\left(\operatorname{RTI}\left(\mathbf{U}_{B} \mid \mathbf{U}_{A}\right)\right)$ if $P\left(\mathbf{U}_{B}>\mathbf{u}_{B} \mid \mathbf{U}_{A}>\mathbf{u}_{A}\right)$ is nondecreasing in each component of $\mathbf{u}_{A}$ for all $\mathbf{u}_{B}$; and $\mathbf{U}_{B}$ is stochastically increasing in $\mathbf{U}_{A}\left(\mathrm{SI}\left(\mathbf{U}_{B} \mid \mathbf{U}_{A}\right)\right)$ if $P\left(\mathbf{U}_{B}>\mathbf{u}_{B} \mid\right.$ $\mathbf{U}_{A}=\mathbf{u}_{A}$ ) is nondecreasing in each component of $\mathbf{u}_{A}$ for all $\mathbf{u}_{B}$. For the sake of simplicity of the next result, we will suppose that the functions $f_{i}$ and the parameter $\theta$ in (2) are non-negative.

Theorem 2.1 Let $\mathbf{U}$ be a random vector whose associated $n$-copula $C_{\theta}$ is defined by (2) and such that the functions $f_{i}, i=1, \cdots, n$, and $\theta$ are nonnegative. Let $\mathbf{U}_{A}$ and $\mathbf{U}_{B}$ be two subsets of $\mathbf{U}$ as in the preceding paragraph. Then:

(i) $\operatorname{LTD}\left(\mathbf{U}_{B} \mid \mathbf{U}_{A}\right)$ if and only if $f_{i}(u) \geq u f_{i}^{\prime}(u)$ for all $u \in[0,1]$ and for every $i \in A$.

(ii) $\operatorname{RTI}\left(\mathbf{U}_{B} \mid \mathbf{U}_{A}\right)$ if and only if $f_{i}(u) \geq(u-1) f_{i}^{\prime}(u)$ for all $u \in[0,1]$ and for every $i \in A$.

(iii) $S I\left(\mathbf{U}_{B} \mid \mathbf{U}_{A}\right)$ if and only if $(-1)^{n} f_{i}^{\prime \prime}(u) \prod_{h \in A-\{i\}} f_{h}^{\prime}\left(u_{h}\right) \geq 0$ for all $i \in A$, and $u, u_{h}$ in $[0,1]$.

Proof. Suppose the non-trivial case $0<u_{i} \leq 1, i=1, \cdots, n$. Since all the $k$-dimensional margins of the $C_{\theta}$ are $\Pi^{k}$ for $2 \leq k<n$, we have that $P\left(\mathbf{U}_{B} \leq \mathbf{u}_{B} \mid \mathbf{U}_{A} \leq \mathbf{u}_{A}\right)=\prod_{k \in B} u_{k}+\theta \prod_{k \in B} f_{k}\left(u_{k}\right) \prod_{j \in A} f_{j}\left(u_{j}\right) / u_{j}$, and $P\left(\mathbf{U}_{B}>\right.$ $\left.\mathbf{u}_{B} \mid \mathbf{U}_{A}>\mathbf{u}_{A}\right)=\prod_{k \in B}\left(1-u_{k}\right)+\theta \prod_{k \in B} f_{k}\left(u_{k}\right) \prod_{j \in A} f_{j}\left(u_{j}\right) /\left(1-u_{j}\right)$. If $f_{i}(u) \geq u f_{i}^{\prime}(u)$ for all $i \in A$ and $u \in(0,1]$, we have $\frac{\partial}{\partial u_{i}} P\left(\mathbf{U}_{B} \leq \mathbf{u}_{B} \mid \mathbf{U}_{A} \leq \mathbf{u}_{A}\right) \leq 0$; and conversely. Thus part (i) holds. A similar argument yields part (ii). Now, since $P\left(\mathbf{U}_{B}>\mathbf{u}_{B} \mid \mathbf{U}_{A}=\mathbf{u}_{A}\right)=\prod_{k \in B}\left(1-u_{k}\right)+(-1)^{|B|} \prod_{k \in B} f_{k}\left(u_{k}\right) \prod_{j \in A} f_{j}^{\prime}\left(u_{j}\right)$, part (iii) follows, which completes the proof.

Let $C_{\theta}$ be an $n$-copula given by $(2)$. If $f_{1}(u)=\cdots=f_{n}(u)$ for all $u$ in $[0,1]$, then $C(\pi \mathbf{u})=C(\mathbf{u})$ for any permutation $\pi$ of $\mathbf{u}$; and if $f_{i}(u)=(-1)^{n} f_{i}(1-u)$ for all $i=1, \cdots, n$ and $u \in[0,1]$, then $C_{\theta}$ is radially symmetric.

We now provide a trivariate example of a 3-copula that belongs to (2).

Example 2.2 Let $f_{i}(u)=u^{b}(1-u)^{a}, 1 \leq i \leq 3$, with $a, b \geq 1$. Then, for all $\left(u_{1}, u_{2}, u_{3}\right)$ in $[0,1]^{3}$, the function

$$
C_{\theta}\left(u_{1}, u_{2}, u_{3}\right)=u_{1} u_{2} u_{3}+\theta u_{1}^{b}\left(1-u_{1}\right)^{a} u_{2}^{b}\left(1-u_{2}\right)^{a} u_{3}^{b}\left(1-u_{3}\right)^{a}
$$


is a 3-copula if and only if

$$
-\min \left\{\frac{1}{\left(K_{a b}^{+}\right)^{2} K_{a b}^{-}}, \frac{1}{\left(K_{a b}^{-}\right)^{3}}\right\} \leq \theta \leq \min \left\{\frac{1}{\left(K_{a b}^{-}\right)^{2} K_{a b}^{+}}, \frac{1}{\left(K_{a b}^{+}\right)^{3}}\right\},
$$

where $K_{a b}^{+}=R^{b-1}(1-R)^{a-1}(R-b), K_{a b}^{-}=S^{b-1}(1-S)^{a-1}(S-b)$, and

$R=\frac{b(a+b-1)+\sqrt{a b(a+b-1)}}{(a+b)(a+b-1)}$ and $S=\frac{b(a+b-1)-\sqrt{a b(a+b-1)}}{(a+b)(a+b-1)}$.

To check this, note that the density of (4) is given by $c_{\theta}\left(u_{1}, u_{2}, u_{3}\right)=1+$ $\theta \prod_{i=1}^{3} u_{i}^{b-1}\left(1-u_{i}\right)^{a-1}\left(b-(a+b) u_{i}\right)$, and the overall constraint for $\theta$ is

$$
\theta \prod_{i=1}^{3} u_{i}^{b-1}\left(1-u_{i}\right)^{a-1}\left(b-(a+b) u_{i}\right) \geq-1 .
$$

It is clear that if $u_{1}=u_{2}=u_{3}=b /(a+b)$, we have that $c_{\theta}\left(u_{1}, u_{2}, u_{3}\right)=1$. Let $g(u)=u^{b-1}(1-u)^{a-1}(b-(a+b) u)$. Then the solutions of $g^{\prime}(u)=0$ are $u_{*}=S<b /(a+b)$ and $u^{*}=R>b /(a+b)$. Furthermore, we have that $g^{\prime \prime}\left(u_{*}\right)<0$ and $g^{\prime \prime}\left(u^{*}\right)>0$. Thus, the function $-g(u)$ (respectively, $g(u)$ ) attains its maximum value at the point $u^{*}$ (respectively, $\left.u_{*}\right)$. Now, consider the orthants $O_{1}=\left\{\left(u_{1}, u_{2}, u_{3}\right) \in[0,1]^{3}: g\left(u_{1}\right)>0, g\left(u_{2}\right)>0\right.$, and $g\left(u_{3}\right)>$ $0\}, O_{2}=\left\{\left(u_{1}, u_{2}, u_{3}\right) \in[0,1]^{3}: g\left(u_{1}\right)<0, g\left(u_{2}\right)<0\right.$, and $\left.g\left(u_{3}\right)<0\right\}$, $O_{3}=\left\{\left(u_{1}, u_{2}, u_{3}\right) \in[0,1]^{3}:\right.$ one of $g\left(u_{1}\right), g\left(u_{2}\right)$ and $g\left(u_{3}\right)$ is positive $\}$ and $O_{4}=\left\{\left(u_{1}, u_{2}, u_{3}\right) \in[0,1]^{3}\right.$ : one of $g\left(u_{1}\right), g\left(u_{2}\right)$ and $g\left(u_{3}\right)$ is negative $\}$. In the orthant $O_{1}$, we have that $\left(u_{1}, u_{2}, u_{3}\right) \in[0, b /(a+b))^{3}$. Hence (5) requires to have $\theta \geq-1 / \prod_{i=1}^{3} u_{i}^{b-1}\left(1-u_{i}\right)^{a-1}\left(b-(a+b) u_{i}\right)$. In this case, the maximum is attained at the point $u_{*}$. Thus $\theta \geq-1 /\left(K_{a b}^{-}\right)^{3}$. In $O_{2}$, we have that $b /(a+b) \leq$ $u_{1}, u_{2}, u_{3}<1$. Hence (5) requires to have $\theta \leq 1 / \prod_{i=1}^{3} u_{i}^{b-1}\left(1-u_{i}\right)^{a-1}\left[(a+b) u_{i}-b\right]$. In this case, the maximum is attained at the point $u^{*}$. Thus $\theta \leq 1 /\left(K_{a b}^{+}\right)^{3}$. In $\mathrm{O}_{3}$, two of $u_{i}^{\prime} s$ are greater than $b /(a+b)$. For instance, if $\left(u_{1}, u_{2}\right) \in(b(a+b), 1)^{2}$ and $0 \leq u_{3}<b /(a+b)$, we are required to have $\theta \geq-1 / u_{3}^{b-1}\left(1-u_{3}\right)^{a-1}[b-$ $\left.(a+b) u_{3}\right] \prod_{i=1}^{2} u_{i}^{b-1}\left(1-u_{i}\right)^{a-1}\left[(a+b) u_{i}-b\right]$. Hence $\theta \geq-1 /\left(K_{a b}^{+}\right)^{2} K_{a b}^{-}$. A similar argument shows that $\theta \leq 1 /\left(K_{a b}^{-}\right)^{2} K_{a b}^{+}$for the orthant $O_{4}$.

Observe that if $a=b=1$, we have a (one-parametric) trivariate extension of the FGM family with $\theta \in[-1,1]$. Moreover, $\tau_{3}\left(C_{\theta}\right)=\rho_{3}\left(C_{\theta}\right)=0$. Let $C_{\theta_{1}}$ and $C_{\theta_{2}}$ be two copulas of the type given by (4). Then $C_{\theta_{1}}$ is more PLOD than $C_{\theta_{2}}$ if $\theta_{1} \geq \theta_{2}$. Finally, suppose $\theta>0$; then, if $\boldsymbol{U}$ is a random vector with 3-copula $C_{\theta}$, from Theorem 2.1 we have that $C_{\theta}$ is $\operatorname{LTD}\left(\mathbf{U}_{B} \mid \mathbf{U}_{A}\right)$ if and only if $b=1, C_{\theta}$ is $\operatorname{RTI}\left(\mathbf{U}_{B} \mid \mathbf{U}_{A}\right)$ if and only if $a=1$, and $C_{\theta}$ is $\operatorname{SI}\left(\mathbf{U}_{B} \mid \mathbf{U}_{A}\right)$ if and only if $a=b=1$. 


\section{$2.2 \quad$ A second family}

Our next purpose is to define a new class of one-parametric $n$-copulas that generalizes (1) and such that the $k$-margins are in the class. Let $f$ and $g$ be two functions as in Section 1. Let $\alpha$ be a real number, and consider the function $C_{\alpha}^{*}$ given by

$$
C_{\alpha}^{*}(\mathbf{u})=\prod_{i=1}^{n} u_{i}+\alpha \sum_{1 \leq i<j \leq n} f\left(u_{i}\right) g\left(u_{j}\right) \prod_{\substack{k=1 \\ k \neq i, j}}^{n} u_{k}, \quad \mathbf{u} \in[0,1]^{n} .
$$

Then each $k$-margin, $2 \leq k<n$, of (6) is again in this family. $C_{\alpha}^{*}$ is an absolutely continuous function with density $c_{\alpha}^{*}(\mathbf{u})=1+\alpha \sum_{1 \leq i<j \leq n} f^{\prime}\left(u_{i}\right) g^{\prime}\left(u_{j}\right)$. Thus, the function defined by (6) is an $n$-copula if and only if

$$
\frac{-1}{\max \left\{\sum_{1 \leq i<j \leq n} f^{\prime}\left(u_{i}\right) g^{\prime}\left(u_{j}\right)\right\}} \leq \alpha \leq \frac{-1}{\min \left\{\sum_{1 \leq i<j \leq n} f^{\prime}\left(u_{i}\right) g^{\prime}\left(u_{j}\right)\right\}} .
$$

The values of Kendall's tau and Spearman's rho associated with $C_{\alpha}^{*}$ are given by $\tau_{n}\left(C_{\alpha}^{*}\right)=2 I_{2} /\left(2^{n-1}-1\right)$ and $\rho_{n}\left(C_{\alpha}^{*}\right)=(n+1) I_{2} /\left[2^{n}-(n+1)\right]$, where $I_{2}=2 \alpha n(n-1) \int_{0}^{1} f(u) \mathrm{d} u \int_{0}^{1} g(u) \mathrm{d} u$. Observe that for this class of $n$-copulas we have (again) the relationship in (3) with $C_{\alpha}^{*}$.

Let $\left\{C_{\alpha}^{*}\right\}$ be the corresponding family of $n$-copulas given by (6). Then, $\left\{C_{\alpha}^{*}\right\}$ is positively ordered if and only if $\sum_{1 \leq i<j \leq n} f\left(u_{i}\right) g\left(u_{j}\right) \prod_{\substack{k=1 \\ k \neq i, j}}^{n} u_{k} \geq 0$.

Let $C_{\alpha_{l}}^{*}(\mathbf{u})=\prod_{i=1}^{n} u_{i}+\alpha_{l} \sum_{1 \leq i<j \leq n} f_{l}\left(u_{i}\right) g_{l}\left(u_{j}\right) \prod_{\substack{k=1 \\ k \neq i, j}}^{n} u_{k}(l=1,2)$ be two $n$ copulas. $C_{\alpha_{1}}^{*}$ is more PLOD than $C_{\alpha_{2}}^{*}$ when $\alpha_{1} \sum_{1 \leq i<j \leq n} f_{1}\left(u_{i}\right) g_{1}\left(u_{j}\right) \prod_{\substack{k=1 \\ k \neq i, j}}^{n} u_{k} \geq$ $\alpha_{2} \sum_{1 \leq i<j \leq n} f_{2}\left(u_{i}\right) g_{2}\left(u_{j}\right) \prod_{\substack{k=1 \\ k \neq i, j}}^{n} u_{k}$. On the other hand, since $\bar{C}_{\alpha}^{*}(\mathbf{u})=\prod_{i=1}^{n}\left(1-u_{i}\right)+$ $\alpha_{1} \sum_{1 \leq i<j \leq n} f_{1}\left(u_{i}\right) g_{1}\left(u_{j}\right) \prod_{\substack{k=1 \\ k \neq i, j}}^{n}\left(1-u_{k}\right)$, a similar result holds for PUOD.

Let $C_{\alpha}^{*}$ be an $n$-copula given by (6). If $f(u)=g(u)$ for all $u$ in $[0,1]$ then $C(\pi \mathbf{u})=C(\mathbf{u})$ for any permutation $\pi$ of $\mathbf{u}$; and if $f(u)=f(1-u)$ and $g(u)=g(1-u)($ or $f(u)=-f(1-u)$ and $g(u)=-g(1-u)), u \in[0,1]$, then $C$ is radially symmetric.

Example 2.3 Let $f$ and $g$ be two functions such that $f(u)=g(u)=u(1-u)$ for all $u \in[0,1]$, and let $\mathbf{a}=\left(a_{1}, \cdots, a_{n}\right)$ be a point in $[0,1]^{n}$ such that $a_{i}= \pm 1$ 
for all $i=1, \cdots, n$. It is easy to check that $\max _{u \in[0,1]} f^{\prime}(u)=\max _{u \in[0,1]} g^{\prime}(u)=1$ and $\min _{u \in[0,1]} f^{\prime}(u)=\min _{u \in[0,1]} g^{\prime}(u)=-1$. Thus, we must obtain the maximum and the minimum value of $\sum_{1 \leq i<j \leq n} a_{i} a_{j}$ for which the inequalities in (7) are satisfied. It is clear that $\max \left(\sum_{1 \leq i<j \leq n} a_{i} a_{j}\right)$ in $Q^{+}=\left\{\mathbf{a} \in[0,1]^{n}: \sum_{1 \leq i<j \leq n} a_{i} a_{j} \geq 0\right\}$ is obtained when $a_{i}=1$ for all $i=1, \cdots, n$ or $a_{i}=-1$ for all $i=1, \cdots, n$, and equal to $n(n-1) / 2$. Therefore $\theta \geq-2 /[n(n-1)]$. For the upper bound of $\theta$, we must find the minimum value of $\sum_{1 \leq i<j \leq n} a_{i} a_{j}$ in $Q^{-}=\left\{\mathbf{a} \in[0,1]^{n}\right.$ : $\left.\sum_{1 \leq i<j \leq n} a_{i} a_{j} \leq 0\right\}$. But $\sum_{1 \leq i<j \leq n} a_{i} a_{j}=(1 / 2) \sum_{i=1}^{n} a_{i} \sum_{\substack{j=1 \\ j \neq i}}^{n} a_{j}$. If $k$ (respectively, $n-k)$ is the number of $a_{i}^{\prime} s$ such that are equal to 1 (respectively, -1), then $\sum_{i=1}^{n} a_{i}=k-(n-k)=2 k-n$. So $\sum_{1 \leq i<j \leq n} a_{i} a_{j}=(1 / 2) \sum_{i=1}^{n} a_{i}\left(2 k-n-a_{i}\right)=$ $(1 / 2)\left[(2 k-n) \sum_{i=1}^{n} a_{i}-\sum_{i=1}^{n} a_{i}^{2}\right]=\left((2 k-n)^{2}-n\right) / 2$, and $\min _{\mathbf{a} \in Q^{-}} \sum_{1 \leq i<j \leq n} a_{i} a_{j}=$ $-\lfloor n / 2\rfloor$, where $\lfloor t\rfloor$ denotes the integer part of the real number $t$. In short, the function $C_{\alpha}^{*}(\mathbf{u})=\prod_{i=1}^{n} u_{i}+\alpha \sum_{1 \leq i<j \leq n} u_{i} u_{j}\left(1-u_{i}\right)\left(1-u_{j}\right) \prod_{\substack{k=1 \\ k \neq i, j}}^{n} u_{k}$ is an n-copula if and only if $-2 /[n(n-1)] \leq \alpha \leq 1 /\lfloor n / 2\rfloor$. The expressions for $\tau_{n}$ and $\rho_{n}$ are given by $\tau_{n}\left(C_{\alpha}^{*}\right)=n(n-1) \alpha /\left[9\left(2^{n-1}-1\right)\right]$ and $\rho_{n}\left(C_{\alpha}^{*}\right)=n\left(n^{2}-1\right) \alpha /\left[18\left(2^{n}-\right.\right.$ $n-1)]$. Furthermore, $C_{\alpha}^{*}$ is radially symmetric; $C_{\alpha}^{*}$ is more POD than $\Pi^{n}$ if and only if $\alpha \geq 0$; and $C_{\alpha}^{*}(\pi \mathbf{u})=C_{\alpha}^{*}(\mathbf{u})$ for any permutation $\pi$ of $\mathbf{u}$.

\subsection{A third family}

Let $\Theta=\left\{\theta_{i_{1} \cdots i_{k}}: 1 \leq i_{1}<\cdots<i_{k} \leq n, 2 \leq k \leq n\right\}$ be a set of $2^{n}-n-1$ parameters. A third class of distributions which generalizes (1) can be the following $\left(2^{n}-n-1\right)$-parameter family:

$$
C_{\Theta}^{* *}(\mathbf{u})=\prod_{i=1}^{n} u_{i}+\sum_{k=2}^{n} \sum_{1 \leq i_{1}<\cdots<i_{k} \leq n} \theta_{i_{1} \cdots i_{k}} \prod_{j=i_{1}}^{i_{k}} f_{j}\left(u_{j}\right) \prod_{\substack{l=1 \\ l \neq i_{1}, \cdots, i_{k}}}^{n} u_{l}, \quad \mathbf{u} \in[0,1]^{n}
$$

where $f_{i}, 1 \leq i \leq n$, are $n$ non-zero absolutely continuous functions such that $f_{i}(0)=f_{i}(1)=0$. Using the density function, we have that $C_{\Theta}^{* *}$ is an $n$-copula if and only if $1+\sum_{k=2}^{n} \sum_{1 \leq i_{1}<\cdots<i_{k} \leq n} \theta_{i_{1} \cdots i_{k}} \prod_{j=i_{1}}^{i_{k}} f_{j}^{\prime}\left(u_{j}\right) \geq 0$ for all $u_{i} \in[0,1], 1 \leq i \leq n$. Each $k$-margin, $2 \leq k<n$, of a member given 
by (8) is again an $k$-copula of the same type. We obtain that $\tau_{n}\left(C_{\Theta}^{* *}\right)=$ $2 I_{3} /\left(2^{n-1}-1\right)$ and $\rho_{n}\left(C_{\Theta}^{* *}\right)=(n+1) I_{3} /\left(2^{n}-n-1\right)$, where $I_{3}=\sum_{k=2}^{n} 2^{k-1}(1+$ $\left.(-1)^{k}\right) \sum_{1 \leq i_{1}<\cdots<i_{k} \leq n} \theta_{i_{1} \cdots i_{k}} \prod_{j=i_{1}}^{i_{k}} \int_{0}^{1} f_{j}\left(u_{j}\right) \mathrm{d} u_{j}$. Again, we have the relationship in (3) with $C_{\Theta}^{* *}$.

Finally, if $f_{i}(u)=-f_{i}(1-u)$ for all $i=1, \cdots, n$ in (8), then we have that $C_{\Theta}^{* *}$ is radially symmetric.

The $n$-copulas given by (8) are a "natural" extension of the (known) FGM family of $n$-copulas (see [5]) taking $f_{j}(u)=u(1-u), u \in[0,1]$, for all $j=$ $1, \cdots, n$ (for applications, see [4]). Denoting this family by $E_{\Theta}^{* *}$, we have that $\tau_{n}\left(E_{\Theta}^{* *}\right)=I_{4} /\left(2^{n}-n-1\right)$ and $\rho_{n}\left(E_{\Theta}^{* *}\right)=(n+1) I_{4} /\left[2\left(2^{n}-n-1\right)\right]$, where $I_{4}=\sum_{k=2}^{n}\left[1+(-1)^{k}\right] / 3^{k} \sum_{1 \leq i_{1}<\cdots<i_{k} \leq n} \theta_{i_{1} \cdots i_{k}}$.

ACKNOWLEDGEMENTS. The first author thanks the research council of Yazd University for support. The second author thanks the support by the Ministerio de Ciencia y Tecnología (Spain) and FEDER, under research project BFM2003-06522.

\section{References}

[1] I. Bairamov and S. Kotz, Dependence structure and symmetry of HuangKotz FGM distributions and their extensions, Metrika, 56 (2002), 55 72 .

[2] I. Bairamov and S. Kotz, On a new family of positive quadrant dependent bivariate distributions, International Mathematical Journal, 3 (2003), 1247 - 1254.

[3] I. Bairamov, S. Kotz and M. Bekçi, New generalized Farlie-GumbelMorgenstern distributions and concomitants of order statistics, Journal of Applied Statistics, 28 (2001), 521 - 536.

[4] D.A. Conway, Farlie-Gumbel-Morgenstern distributions, in: Encyclopedia of Statistical Sciences, Vol. 7 (N.L. Johnson and S. Kotz, editors), John Wiley \& Sons, New York, 1983, pp. 28 - 31.

[5] N.L. Johnson and S. Kotz, Distributions in Statistics: Continuous Multivariate Distributions, John Wiley \& Sons, New York, 1972.

[6] R.B. Nelsen, An Introduction to Copulas, Springer, New York, 1999. 
[7] R.B. Nelsen, Concordance and copulas: A survey, in: Distributions with Given Marginals and Statistical Modelling (C. Cuadras, J. Fortiana and J.A. Rodríguez, editors), Kluwer Academic Publishers, Dordrecht, 2002, pp. $169-178$.

[8] J.A. Rodríguez-Lallena and M. Úbeda-Flores, A new class of bivariate copulas, Statistics \& Probability Letters, 66 (2004), 315 - 325.

Received: April 28, 2005 